\title{
Review of Leaf Unhealthy Region Detection Using Image Processing Techniques
}

\author{
S. A. Dhole ${ }^{1}$, Rukaiyya P. Shaikh ${ }^{{ }^{2}}$ \\ Department of Electronics \& Telecommunication, Bharati Vidyapeeth College of Engineering for Women, \\ Pune, 43 Savitribai Phule Pune University, India \\ ${ }^{*}$ Corresponding author, email: Sampadadhole@rediffmail.com ${ }^{1}$, rukaiyyashaikh1993@gmail.com²
}

\begin{abstract}
In agricultural field the plants comes to an attack from the various pets bacterial and microorganism diseases. This diseases attacks on the plant leaves, steams, and fruit part. This present review paper discussed the image processing techniques which is used in performing the early detection of plant diseases through leaf feature inspection. the basic objective of this work is to develop image analysis and classification techniques for extraction and finally classified the diseases present on leaf. Image of leaf is captured and the process is performed and to determine the status of each plant. Here proposed model divide into four parts. The image preprocessing including normalization and contrast adjustment; segment the region of interest determine by using color transform $\mathrm{YCbCr}$ and bi-level thresholding for statistical usage to determine the defect and severity area of plant leaves. The texture feature extraction using statistical GLCM (Gray Level Co-occurrences Matrix) and color feature by means values. [1] Finally classification achieved using random markov model
\end{abstract}

Keywords: image, leaf, signal

\section{Introduction}

Agriculture is backbone of our India and our economy depends upon agriculture field. Indian farmers have wide range of diversity to select proper fruit and Vegetable crops. The cultivation of these crops for optimum yield \& quality product is highly technical. The fruit crop requires frequent monitoring and application of pesticides to protect the crop till harvesting. Diagnosis of Plant diseases are more vital to prevent losses in quality \& quantity of product. Leaf diseases can be diagnosed at early stage which helps to protect not only the leaves but fruits also. farmers normally monitors the growth \& condition of crop right from the Plantation. To protect the crops from diseases farmers are applying chemicals without knowing requirement of plants, hence diagnosis and timely protection of plant are more important.

\section{Related Work}

Ms. Kiran R. Gavhale et al. [1] have presents a number of image processing techniques and it is helpful to extract diseased part of leaf. For color image enhancement purpose SF-CES technique are used. For disease part extraction the $\mathrm{K}$ mean clustering used and $\mathrm{YCbCr}$ color space supports $\mathrm{K}$ mean clustering. For further classification purpose the GLCM texture feature and color texture features are extracted. And finally classification is done by based on SVM.

Jiangsheng Gui et al. [2] have proposed a novel method to extract crop disease region based on saliency detection. This is very easy to implement and noise tolerant. This paper compared with original Itti method and new method to determine salient region in image. For this purpose using low-level features of luminance and color in CIELab color space. This combined with multi scale analysis by changing the size of each scale of the filter. Finally then generated the salience map. This method used low-level features of luminance and color and it combined with multi-scale analysis to determine saliency maps in images, and then Fuzzy Kmeans (USFCM) clustering algorithm was used.

Rittika Raichaudhuri et al.[3] this project is to provide a system for detecting wheat leaf diseases. In this automated system will be used to detect the wheat leaf diseases though image 
processing. The $\mathrm{k}$ mean algorithm and canny filter are usage for the image processing and segmentation processing. Pattern recognition is achieved through PCA or GLCM and classification through SVM or ANN. Techniques such as median filter, histogram equalization, image smoothening, image sharpening, etc., can be used for performing image enhancement. Some of the algorithm techniques that can be used for texture feature extraction are gabor filter, color co-occurrence methods, wavelet transform, etc.

Amar Kumar Dey et al. [4] have implemented Otsu thresholding method. This method is based on image processing algorithm for segmentation of leaf rot diseases. The proposed vision based method are efficient in detecting and observing the exterior diseases features. This methodology has three stages. The initial stage was the image acquisition stage. Initially the real word sample is recorded in its digital form using flat bed digital scanner. Then in next stage the research image was subjected to a preprocessing stage. Size and complexity is completely reduced in this stage. Finally image processing algorithms calculated the area of the segmented part of leaf.

\section{Summary}

Previous papers are described different techniques to diagnose leaf diseases. We should make further research on test equipment, image processing and analysis method to achieve better detection accuracy with different plant species.

\section{Proposed Methodology}

Figure 1 shows diagram of proposed methodology. Step proposed methodology:

a. Image Acquisition

The image of various leaves of citrus plant like grapefruit, limes, lemon, orange is collected using a digital camera. The input photo image is resized for further operation.

b. Image Preprocessing

The lowest level of abstraction is the image preprocessing and its aim is to improve the image data. This is helpful to suppress undesired distortion and enhances some image features. This features is important for further processing and analysis task. It includes normalization and contrast adjustment.

c. Image Segmentation

Segmentation is process used to simplify the representation of an image into something that is more meaningful object of interest from background. Segmentation is also done through color transform into $\mathrm{YCbCr}$ and bi-level thresholding.

d. Feature Extraction

After segmentation the area of interest mean actually diseased part extracted. The aim of this phase is to find and extract features of a given sample. Image features usually include color, shape and texture features in image processing. The Texture is one of most popular features used for image classification. Currently most of the researchers using plant leaf texture and it is the most important feature in classifying plants

e. Classification

In classification it construct a hyper plane in a high dimensional space and it helpful for used for classification. The hyper plane is used for good separation that has the largest distance between to the nearest training data points. In classification and recognition randomly divide database for training and testing 


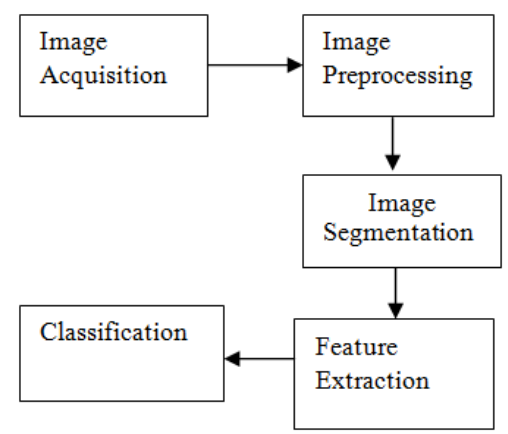

Figure 1. Block diagram of proposed methodology [1]

\section{Conclusion}

This paper review process application of image processing diagnose leaf diseases. By implementation of this technique we can minimize use of harmful chemicals on plants which in turn gives healthier environment. We should further research and implement the digital image processing for better detection of plant diseases.

\section{References}

[1] Ms Kiran R, Gavhale, Mr Kamal O Hajari. Unhealthy Region of Citrus Leaf Detection Using Image Processing Techniques. International Conference for Convergence of Technology. 2014.

[2] Jiangsheng Gui, Li Hao, Qing Zhang, Xiaoan Bao. New Method for Soybean Leaf Disease Detection Based on Modified Salient Regions. International Journal of Multimedia and Ubiquitous Engineering. 2015; 10(6):45-52. http://dx.doi.org/10.14257/ijmue. 2015.10.6.06.

[3] Rittika Raichaudhuri, Rashmi Sharma. On Analysis of Wheat Leaf Infection by Using Image Processing. Proceedings of the International Conference on Data Engineering and Communication Technology. Advances in Intelligent Systems and Computing 468. DOI 10.1007/978-981-10-16752_56.

[4] Amar Kumar Deya, Manisha Sharmaa, MR Meshramb. Image Processing Based Leaf Rot Disease, Detection of Betel Vine. International Conference on Computational Modeling \& Security (CMS 2016) Procedia Computer Science 85. 2016: 748-754.

[5] RM Haralick, K Shanmugam, D Its'shak. Textural Features for Image Classification. Systems, Man and Cybernetics, IEEE Transactions on. 1973; 3(6): 613-621.

[6] Anand H. Kulkarni, Ashwin Patil RK. Applying image processing technique to detect plant diseases. International Journal of Modern Engineering Research. 2012; 2(5): 3661-3664.

[7] Vijai Singh, Prof AK Misra. Detection of unhealthy region of plant leaves using Image Processing and Genetic Algorithm. International Conference on Advances in Computer Engineering and Applications (ICACEA) IMS Engineering College, Ghaziabad, India. 2015.

[8] Vijai Singh, Prof AK Misra. Detection of unhealthy region of plant leaves using Image Processing and Genetic Algorithm. International Conference on Advances in Computer Engineering and Applications (ICACEA) IMS Engineering College, Ghaziabad, India. 2015.

[9] Akanksha Singh, Sini Shibu, Shatendra Dubey. Recent Image Enhancement Techniques: A Review. International Journal of Engineering and Advanced Technology (IJEAT). 2014; 4 (1).

[10] Dheeb Al Bashish, Malik Braik, and Sulieman Bani-Ahmad. A Framework for Detection and Classification of Plant Leaf and Stem Diseases. International Journal of Modern Engineering Research. 2012; 2(5): 3661-3664. 